\title{
Legg-Calvé-Perthes hastalığı sekellerinde total kalça artroplastisi tedavisi ve sonuçları
}

\section{Results of total hip arthroplasty for the sequelae of Legg-Calvé-Perthes disease}

\author{
Ömür Çağlar \\ Hacettepe Universitesi Tıp Fakültesi, Ortopedi ve Travmatoloji Anabilim Dalı, Ankara
}

\begin{abstract}
Legg-Calvé-Perthes hastalığı sonrasında doğal olarak, uygun tedaviye rağmen rölatif olarak erken yaşlarda sekonder kalça osteoartriti gelişebilmektedir. Total kalça protezi, Legg-Calvé-Perthes hastalığı sonrasında gelişen sekellerin tedavisinde etkin ve güvenilir bir seçenektir. Legg-Calvé-Perthes hastalığı sonrasında uygulanan konvansiyonel çağdaş total kalça artroplastisi sonuçları, primer osteoartrit sonrası uygulamalara benzer sonuçlar göstermektedir. Ancak literatüre göre, geniş serilerde, total artroplasti sonrasında peri-prostetik kırık ve nörolojik komplikasyon oranları yüksektir. Tipik olarak görülen rezidüel koksa breva deformitesi; kısa femur boynu, geniş oval femur başı, rölatif olarak uzamış trokanter major ve azalmış baş-boyun açısını içerir. Bununla beraber, femur diyafizi çoğu zaman etkilenmez ve klasik protezlerin yerleştirilmesine izin verir. Asetabulum ise çoğu zaman sığ, konkavitesini kaybetmiş ve femur başına uyum sağlamıştır. Ancak, mediyal duvar primer asetabular komponent için çoğu zaman yeterli kalınlıktadır. Bazı yazarlar, modüler veya özel yapım protezlerin kullanılmasını savunmuşlardır. Burada özellikle, metafiz ile diyafiz arasındaki boyut ve şekil uyumsuzluğu hedeflenmektedir. Bu serilerin sonuçları da, primer osteoartrit sonrası total kalça protez uygulamalarına benzerdir.
\end{abstract}

Anahtar sözcükler: Legg-Calvé-Perthes hastalığı; total kalça artroplastisi; komplikasyon
Despite appropriate treatment, secondary hip osteoarthritis may develop after Legg-Calvé-Perthes disease at a relatively younger age. Total hip arthroplasty is safe and efficient method for the treatment of sequelae after LeggCalvé-Perthes disease. Implementation of today's conventional total hip arthroplasty after Legg-Calvé-Perthes disease produces similar success rates as total hip arthroplasty after primary osteoarthritis of the hip. However, according to literature, larger series report higher rates of neurologic complications and peri-prosthetic fractures. Residual coxa breva, a typical deformity seen after LeggCalvé-Perthes disease involves short femoral neck, relatively overgrown trochanter major, larger femoral head and decreased neck-shaft angle. However, the femoral diaphysis is often unaffected and allows the placement of conventional prostheses. On the other hand, the acetabulum is often shallow, has lost its concavity and adapted to the femur. But it has enough medial wall for placement of a hemispherical component. Some reports suggest using modular or custom made components. Specifically, the size and shape mismatch between metaphysis and diaphysis is aimed at here. The results of these series are also similar to those of total hip prosthesis after primary osteoarthritis.

Key words: Legg-Calvé-Perthes disease; total hip arthroplasty; complications
egg-Calvé-Perthes hastalığı (LCPH), femur başında geçici olarak kan akımının kesilmesi ve osteonekrozun gelişmesine neden olan idiyopatik çocukluk çağı hastalığıdır. Uzun dönem tedavi sonuçları iyi değildir ve doğal seyrin tam olarak çözülememiş olması da hastalığın tedavisini güçleştirmektedir. Bununla beraber, breys, immobilizasyon ve yatak istirahatinden femoral veya asetabular osteotomilere kadar, pek çok tedavi seçeneği kullanılmaktadır.
Gelişen osteonekroz, herhangi bir kemik deformitesi bırakmadan iyileşebileceği gibi, proksimal femur ve kalça ekleminde belirgin deformite ve osteoartrit ile de sonuçlanabilir. İskemik nekroz, femur başını mekanik olarak zayıflatır ve izleyen yüklenme, femur başında deformitelere neden olur. ${ }^{[1]}$ Asetabular değişiklikler, gelişimsel kalça displazisine benzer şekilde, çoğu zaman femoral deformiteyi izleyen yeniden şekillenme sonrasında olur. Erken ve orta dönem

- İletişim adresi: Doç. Dr. Ömür Çağlar, Hacettepe Universitesi Tıp Fakültesi, Ortopedi ve Travmatoloji Anabilim Dalı, Sıhhiye, Ankara Tel: 0312 - 3051209 e-posta: ocaglar@hacettepe.edu.tr

- Geliş tarihi: 20 Şubat $2017 \quad$ Kabul tarihi: 20 Şubat 2017 
izlemlerde geride kalan deformiteler iyi tolere edilse de, yaşamın 6 . onyılında hastaların yaklaşık olarak \%50'sinde belirgin olarak artrit gelişmektedir. ${ }^{[2]}$ Olguların bir kısmında, tedaviden bağımsız olarak ikincil osteoartrit gelişir.

Yakın zamanda yayımlanmış bir makalede, cerrahidışı LCPH tedavisi sonrasında \%5 oranında total kalça artroplastisi rapor edilmiştir. ${ }^{[3]}$ Bununla beraber, Perthes hastalığı sonrasında total kalça artroplastisi ile ilgili literatür kısıtıdır. Optimal tedavi yöntemleri geliştirilmesi için, LCPH sonrasında uygulanan artroplastide sağkalım üzerinde etkili faktörlerin ve risklerin tanımlanması gerekmektedir.

Bu makalede, LCPH sonrasında son dönem osteoartrit gelişen hastalarda uygulanan artroplasti tekniğindeki farklılıklar ve literatürdeki az sayıda serinin sonuçları tartışılacaktır.

\section{LCPH SEKELLERINDE ARTROPLASTI}

LCPH sonrası gelişen sekellerde sık karşılaşılan kemik anatomisi değişiklikleri, total kalça protezi planlanması öncesi dikkate alınmalıdır. Bunlardan ilki, femoral metafizyel ve diyafizyel bölge morfolojisinde oluşan değişikliklerdir. Çoğu zaman, iki bölge arasında boyut ve anatomi açısından uyumsuzluk vardır. Bu da, piyasada bulunan modern çimentosuz pressfit protezlerin, normale göre teknik olarak zor uygulanmasını veya özel yapım protezler ile modüler protezlerin kullanımını gündeme getirmiştir. Hastaların bir kısmında, displastik proksimal femur anatomisini düzeltici osteotomilere ihtiyaç duyabilir. ${ }^{[4]}$ Ameliyat öncesinde, trokanterik yüksekliğin rölatif olarak artması, anormal femur boyun uzunluğu ve pozisyonu ${ }^{[5-7]}$; hastaların pek çoğunda mevcut alt ekstremite eşitsizliği dikkate alınmalıdır. Anormal asetabular gelişme, asetabular yetmezlik ile sonuçlanabilir. Bazı çalışmalarda, asetabulumun deforme olan femur başına göre şekilleneceği ve tüm kalça hareketlerinde olmasa da kabul edilebilir bir uyum sağlayacağı gösterilmiştir. Anormal asetabular gelişmenin, femoral anteversiyon bozukluklarıyla ilişkili olduğu da ileri sürülmüştür..$^{[2,6]}$ Asetabulum bu olgularda tipik olarak sığ, genişlemiş ve retrovert olarak karşımıza çıkar. ${ }^{[8]}$

Genç yaş ve multi-planar deformitelerin varlı̆̆ına ek olarak, çocukluk çağında geçirilmiş olan özellikle femoral ve bunun yanında asetabular osteotomiler de, uygulanacak total kalça artroplastisini komplike hale getirebilir. Geçirilmiş cerrahiler, ameliyat sırasında açıIım zorluklarına, çıkarılan implantların oluşturabileceği kemik defektleri neticesinde peri-prostetik kırıklara neden olabilir. ${ }^{[9]}$

\section{TOTAL KALÇA PROTEZi TEKNIĞi}

Koksa breva, LCPH sonrasında gelişen tipik bir deformitedir. Kısa femur boynu; geniş, oval femur başı, rölatif olarak uzamış büyük trokanter ve azalmış baş boyun açısı tipiktir. Çoğu zaman, büyük femur başı laterale doğru sublukse olur ve asetabulum tarafindan yeterince kapsanmaz. İntertrokanterik bölge distal kesimi ve diyafiz ise çoğu zaman normaldir. Bu nedenle, çoğu kez aslında klasik femoral stem yerleştirilebilir. Femur başı ve boynunun ciddi olarak deforme olduğu hastalarda femur boyun kesisi, uygun ekstremite boyunu sağlamak için çoğu zaman başın ortasından yapılmalıdır. Asetabulum mediyal duvarı çoğu zaman standart bir component örtünmesi için yeteri kadar kalındır ve modern asetabular komponentler kolaylıkla yerleşir. ${ }^{[10]}$

Legg-Calvé-Perthes hastalığı sekelleri sonrasında total kalça protez sonuçlarını inceleyen literatürde az sayıda makale mevcuttur. Bununla beraber genel olarak yazarlar, hem cerrahi sırasında yaşanabilecek güçlüklerden ve özel protez gereksiniminden hem de sık görülebilecek olası komplikasyonlardan söz etmektedirler (Şekil 1).

\section{TOTAL KALÇA PROTEZI SONUÇLARI}

Bu konuda en geniş serilerden biri, Baghdadi ve arkadaşları tarafından yayımlanmıştır. Çocukluk çağında LCPH tanısı almış ve dokümantasyonu bulunan 95 hastanın 99 kalçası, minimum iki yıl takip, ortalama $8 \pm 5$ yıl olacak şekilde değerlendirilmiştir. Hastaların ortalama yaşları $48 \pm 15$ 'tir. Yazarlar, toplamda 10 adet revizyon bildirmişlerdir. Sekiz yıllık sağkalımları çimentosuz implantlar için \%90, hybrid implantlar için \%86 olarakve tüm hastalarda kalça skorlarında anlamlı artış rapor edilmiştir. ${ }^{[9]}$ Yazarlar, göreceli olarak yüksek oranda komplikasyon bildirmişler; en sık ameliyat sırasında kırık ve nörolojik komplikasyonları rapor etmişler ve fazla boy uzatmadan kaçınılmasını önermişlerdir. Serilerinde, cerrahi sırasında sekiz femoral ve bir asetabular kırık gelişmiştir (\%3). Literatürde bu oran yaklaşık olarak \%0,17 olarak belirtilmektedir. Üç adet de siyatik sinir arazı rapor edilmiştir. Illginç olarak, bu hastalarda ameliyat sonrasında ortalama 1,2 $\pm 0,4 \mathrm{~cm}$ uzama olmuştur. Ameliyat sırasında üç hastada, rutin yaklaşımlar dışında açılım için trokanterik osteotomiye ihtiyaç duymuşlardır. Geçirilmiş çocukluk çağı cerrahi öyküsü olan hastalarda, istatistiksel olarak anlamlı olmasa da, 12. yıl takiplerinde revizyon oranı daha yüksek olarak rapor edilmiştir. ${ }^{[9]}$

Seufert ve arkadaşları ise, modüler kalça protezi kullanarak tedavi ettikleri serilerinde 28 hastanın $35 \mathrm{kal}-$ çasını değerlendirmişler, sadece bir hastada geç hemotolojik enfeksiyon dışında revizyon bildirmemişlerdir. 


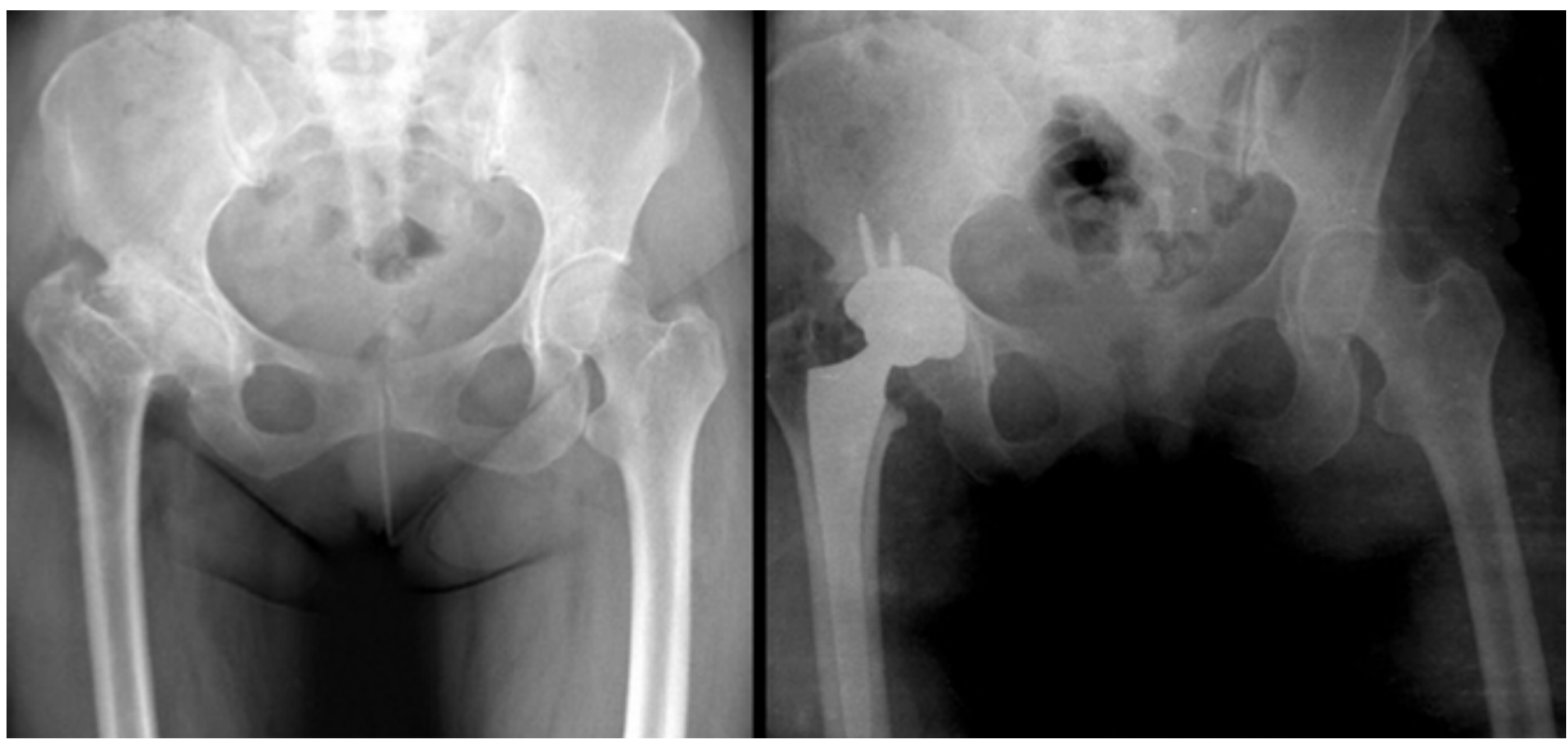

Şekil 1. Kırk dört yaşında bayan hasta, LCPH nedeniyle çocukluk çağında ameliyat olmuştur. Hastanın ameliyat öncesi grafisinde, tipik koksa breva deformitesi görülmektedir. Hasta, proksimali hidroksiapatit kaplı standart bir femoral stem ve hemisferik asetabular komponenti içeren total kalça protezi ile, başarılı olarak tedavi edilmiştir.

Minimum iki yıl ve ortalama 8,2 yıllık takip oranları ile, aslında literatüre göre orta dönemde göreceli olarak başarılı bir seri sunmuşlardır. Modüler implant kullanımının, metafizo-diyafizyel uyumsuzluk olduğunda (serilerinde olguların \%80'i) mükemmel bir çözüm olduğunu belirtmişlerdir. Metafiz ve diyafiz arasında boyut uyumsuzluğu olduğunda, çimentosuz komponentin optimum olarak tespit edilmesinin güç olduğunu ve bir büyük komponentin dar fakat korteksi kalın femurlara kırıklara ya da bir küçük komponentin erken gevşemeye neden olabileceğini belirtmişlerdir. Yine, modüler implantlar sayesinde, mevcut olan anteversiyon kusurunu (olguların \%71'inde) kolaylıkla çözebildiklerini ve bunun da ameliyat sonrası impingement ve instabiliteye çözüm olabileceğini rapor etmişlerdir. Sonuç olarak yazarlar, modüler implantların orta dönem takiplerinde mükemmel sonuç verdiğini, ancak serilerinin küçük olduğunu rapor etmişlerdir. ${ }^{[1]}$

Al-Khateeb ve arkadaşları ise LCPH sekellerinde uyguladıkları özel yapım protez sonuçlarını yayımlamışlar; 14 hastada, 15 kalçaya proksimal femurda deformite nedeniyle uyguladıkları kalça artroplastisini değerlendirmişlerdir. Ortalama yaşları 32,8 olan hastaların ortalama 10 yıllık takip sonuçları değerlendirilmiş; femoral stemlerde on yıllık sağkalım oranı \%100, asetabular komponentte ise $\% 79$ olarak rapor edilmiş; üç asetabular komponent ortalama olarak 8,3 yılda revize edilmiştir. Yazarlar, tüm bu gevşemeleri eski nesil polietilen ve kobalt krom femur başı kullanımına bağlamışlardır.

Traina ve arkadaşları ise, uzun dönem takip ettikleri LCPH sekellerinin total kalça protezi sonuçlarında 27 hastanın 32 kalçasını incelemişlerdir. Ameliyat sırasında ortalama yaşları 37,8 olan hastaların ortalama takip süreleri 10 yıl olarak rapor edilmiş; tüm hastalar içinde yalnızca bir hastada revizyon femoral komponente uygulanmış; iki hastada kalıcı siyatik sinir arazı gelişmiştir. Bir hastada ortalama $27 \mathrm{~mm}$ uzama varken, diğer hastada $7 \mathrm{~mm}$ uzama miktarı bulunmuştur. Yazarlar, uzun dönem takipli serileri sonrasında, LCPH hastalarında standart kalça protezleri kullanarak oldukça iyi uzun dönem sonuçlar alınabileceğini; aynı zamanda, sığ asetabulumda çoğu zaman mediyalizasyona ihtiyaç duyulacağını ve büyük boy komponentler gerekebileceğini belirtmişlerdir. [11]

\section{SONUÇ}

LCPH sonrasında doğal veya iyatrojenik olarak gelişen deformiteler, kalça protezi ameliyatını teknik olarak zorlaştırabilir; bununla beraber, dikkatli bir cerrahi teknik ile çoğu zaman uygulanan artroplasti sonuçları benzer yaşta uygulanan primer osteoartrit grubuna benzerdir. 


\section{KAYNAKLAR}

1. Seufert CR, McGrory BJ. Treatment of Arthritis Associated With Legg-Calvé-Perthes Disease With Modular Total Hip Arthroplasty. J Arthroplasty 2015;30(10):1743-6. Crossref

2. Stulberg SD, Cooperman DR, Wallensten R. The natural history of Legg-Calvé-Perthes disease. J Bone Joint Surg Am 1981;63(7):1095-108.

3. Larson AN, Sucato DJ, Herring JA, Adolfsen SE, Kelly DM, Martus JE, Lovejoy JF, Browne R, Delarocha A. A prospective multicenter study of Legg-Calvé-Perthes disease: functional and radiographic outcomes of nonoperative treatment at a mean follow-up of twenty years. J Bone Joint Surg Am 2012;94(7):584-92. Crossref

4. Al-Khateeb $\mathrm{H}$, Kwok IH, Hanna SA, Sewell MD, HashemiNejad A. Custom cementless THA in patients with LeggCalvé-Perthes Disease. J Arthroplasty 2014;29(4):792-6. Crossref

5. Mattingly DA. The S-ROM modular femoral stem in dysplasia of the hip. Orthopedics 2005;28(9 Suppl):s1069-73.
6. Froberg L, Christensen F, Pedersen NW, Overgaard S. Radiographic changes in the hip joint in children suffering from Perthes disease. J Pediatr Orthop B 2012;21(3):220-5. Crossref

7. Grzegorzewski A, Synder M, Kozlowski P, Szymczak W, Bowen RJ. Leg length discrepancy in Legg-Calvé-Perthes disease. J Pediatr Orthop 2005;25(2):206-9.

8. Sankar WN, Flynn JM. The development of acetabular retroversion in children with Legg-Calvé-Perthes disease. J Pediatr Orthop 2008;28(4):440-3. Crossref

9. Baghdadi YM, Larson AN, Stans AA, Mabry TM. Total hip arthroplasty for the sequelae of Legg-Calvé-Perthes disease. Clin Orthop Relat Res 2013;471(9):2980-6. Crossref

10. Kim J, Cho YJ, Kim HJ. Role of total hip arthroplasty and resurfacing in Legg-Calvé-Perthes disease. J Pediatr Orthop 2011;31(2 Suppl):S241-4. Crossref

11. Traina $F$, De Fine $M$, Sudanese A, Calderoni PP, Tassinari $E$, Toni A. Long-term results of total hip replacement in patients with Legg-Calvé-Perthes disease. J Bone Joint Surg Am 2011;93(7):e25. Crossref 